\title{
Energy-Aware Cognitive Radio Systems
}

\author{
Ebrahim Bedeer, Osama Amin, Octavia A. Dobre, and Mohamed H. Ahmed
}

\begin{abstract}
The concept of energy-aware communications has spurred the interest of the research community in the most recent years due to various environmental and economical reasons. It becomes indispensable for wireless communication systems to shift their resource allocation problems from optimizing traditional metrics, such as throughput and latency, to an environmental-friendly energy metric. Although cognitive radio systems introduce spectrum efficient usage techniques, they employ new complex technologies for spectrum sensing and sharing that consume extra energy to compensate for overhead and feedback costs. Considering an adequate energy efficiency metric - that takes into account the transmit power consumption, circuitry power, and signaling overhead-is of momentous importance such that optimal resource allocations in cognitive radio systems reduce the energy consumption. A literature survey of recent energy-efficient based resource allocations schemes is presented for cognitive radio systems. The energy efficiency performances of these schemes are analyzed and evaluated under power budget, co-channel and adjacentchannel interferences, channel estimation errors, quality-of-service, and/or fairness constraints. Finally, the opportunities and challenges of energy-aware design for cognitive radio systems are discussed.
\end{abstract}

Ebrahim Bedeer

University of British Columbia, 3333 University Way, Kelowna, BC, Canada, V1V 1V7

e-mail: ebrahim.bedeer-mohamed@ubc.ca

Osama Amin

King Abdullah University of Science and Technology, Thuwal, Makkah Province, Saudi Arabia e-mail: osama.amin@kaust.edu.sa

Octavia A. Dobre

Memorial University of Newfoundland, St. Johns, NL, A1B 3X5, Canada

e-mail: odobre@mun.ca

Mohamed H. Ahmed

Memorial University of Newfoundland, St. Johns, NL, A1B 3X5, Canada

e-mail: mhahmed@mun.ca 


\section{Introduction}

Cognitive radio (CR) can considerably enhance the spectrum utilization efficiency by dynamically sharing the spectrum between licensed/primary users (PUs) and unlicensed/secondary users (SUs) [28]. This is achieved by granting SUs opportunistic access to the white spaces within PUs spectrum, while controlling the interference to PUs, i.e., overlay approach [28]. Alternatively, the SUs and PUs may coexist in the same spectral band, i.e., underlay approach [28]. In other words, an overlay coexistence scenario holds if the SUs are allowed to only access the vacant PUs frequency bands. On the other hand, an underlay coexistence scenario holds if the SUs can access occupied PUs frequency bands while meeting the PUs interference thresholds. The performance of overlay approaches is limited by the mutual interference between SUs and PUs, while co-channel interference places stringent power transmission limitations on the SUs transmission in underlay approaches.

In the past few years, energy-aware communications receive a lot of attention from research and industrial communities due to the rising energy costs to operate future wireless networks, ecological, and environmental reasons [15, 23]. Hence, designing energy-aware CR systems is important to improve both the energy and spectrum efficiencies. The performance of the energy-aware CR systems can be improved by properly allocating the available resources while meeting the imposed constraints, e.g., transmit power, interference, quality-of-service (QoS), and/or fairness constraints. A major difference between CR and conventional systems is the capability of the $\mathrm{CR}$ to sense the surrounding wireless environment. In fact, the available resources include transmit power and/or subchannels as in conventional wireless systems in addition to other parameters related to the sensing process such as sensing, transmission, and idle durations; number of SUs sensing the PUs spectrum; and/or SUs assignment to sense the channel.

The available PUs spectrum can be sensed using SUs independently, i.e., single node sensing; however, the performance is degraded by the hidden node problem ${ }^{1}$ [50]. One way to improve the performance of single node sensing is to use cooperative sensing where multiple SUs may cooperate to sense the spectrum [50]. Cooperative sensing shows better sensing performance at the cost of increasing the consumed energy in the sensing process.

The sensing process can be done periodically, i.e., at the beginning of each frame, then the SU decides about its transmission based on the result of the sensing process. For example, if the sensing outcome is that the PU is not using the channel, then the SU can transmit; otherwise, the SU has to wait until the next frame to repeat the process [31]. Another possible approach for spectrum sensing is the sequential approach where the SU senses the channel sequentially and each time after sensing the SU has to make a decision on whether to continue to sense the next channel or to start transmission [39]. This approach is efficient as the SU does not wait for

${ }^{1}$ PUs may not be detected correctly due to many reasons including shadowing, deep fading, and/or location. 


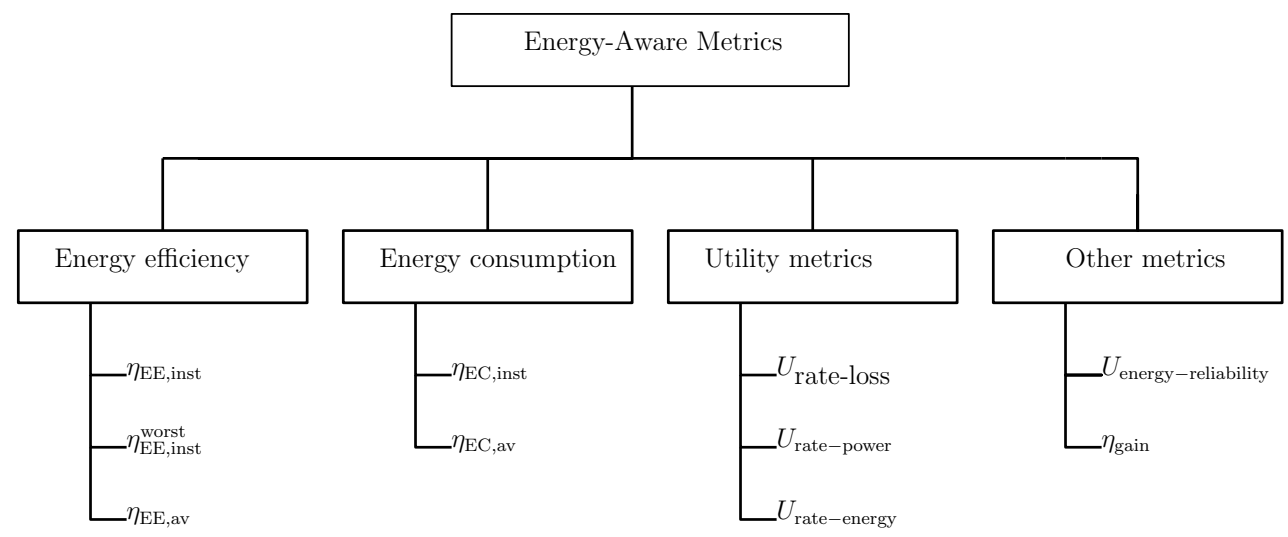

Fig. 1 Summary of EE-aware metrics.

the next frame if the PU channel is not available; however, this is at the cost of consuming more energy in the sensing process.

In this chapter, we provide an overview of the resource allocation problems in energy-aware CR systems. In Section 2 we summarize the commonly used energyaware metrics for CR systems. Section 3 discusses the parameters affecting the resource allocation in energy-aware CR systems. Finally, future trends in energyaware design are discussed in Section 4.

\section{Energy-Aware Metrics}

Different energy-aware metrics are used to quantify the energy efficiency (EE) of $\mathrm{CR}$ systems through considering various parameters that affect $\mathrm{EE}$. A presentation of such metrics is provided in the following sub-sections and summarized in Fig. 1. For the reader's convenance, all the symbols are defined when they first appear, as well as in Tables 1 and 2.

\subsection{Energy efficiency (EE) metrics}

The instantaneous EE is widely used when perfect channel-state-information (CSI) is available. This is defined as the instantaneous transmission rate (or throughput) divided by the instantaneous transmit and consumed circuitry power (or energy), and is expressed in bits/joule (bits/J) [10, 12, 25, 34, 35, 44, 45, 49].

For instance, for multicarrier communication systems, if a single SU employs an orthogonal frequency division multiplexing (OFDM) in order to access the available spectrum, the instantaneous EE is defined as [35, 45] 


$$
\eta_{\mathrm{EE}, \mathrm{inst}}=\frac{B \sum_{n=1}^{N} \log _{2}\left(1+\gamma_{n} p_{n}\right)}{\frac{1}{\zeta} \sum_{n=1}^{N} p_{n}+p_{\mathrm{c}}} \quad(\text { bits } / \mathrm{J})
$$

where $B$ is the subcarrier bandwidth, $N$ is the total number of subcarriers, $\zeta$ is the power amplifier efficiency, $\gamma_{n}$ is the channel-to-interference-plus-noise ratio (CINR) for subcarrier $n, p_{n}$ is the power allocated to subcarrier $n$, and $p_{\mathrm{c}}$ is the circuit power consumption. In case of imperfect CSI on the link between the SU transmitter and receiver, the instantaneous EE is defined as [12]

$$
\eta_{\mathrm{EE}, \text { inst }}=\frac{B \sum_{n=1}^{N} \log _{2}\left(1+\left(\left|\hat{H}_{n}\right|^{2} G p_{n}\right) /\left(\sigma_{\Delta \mathrm{H}}^{2} G p_{n}+\sigma_{n}^{2}+\mathscr{J}_{n}\right)\right)}{\frac{1}{\zeta} \sum_{n=1}^{N} p_{n}+p_{\mathrm{c}}} \quad(\mathrm{bits} / \mathrm{J})
$$

where $\hat{H}_{n}$ is the estimate of the complex channel gain on subcarrier $n, \sigma_{\Delta \mathrm{H}}^{2}$ is the minimum mean square error of the channel estimate, $G$ is the path loss between the SU transmitter and receiver, $\sigma_{n}^{2}$ is the noise variance, and $\mathscr{J}_{n}$ is the interference from all the PUs to subcarrier $n$. On the other hand, if multiple SUs employ orthogonal frequency division multiple access (OFDMA) as the access technique to the available spectrum, then the instantaneous EE is defined as [44]

$$
\eta_{\mathrm{EE}, \text { inst }}=\frac{B \sum_{k=1}^{K} \sum_{n=1}^{N} \rho_{k, n} \log _{2}\left(1+\gamma_{k, n} p_{k, n}\right)}{\frac{1}{\zeta} \sum_{k=1}^{K} \sum_{n=1}^{N} \rho_{k, n} p_{k, n}+p_{\mathrm{c}}} \quad(\mathrm{bits} / \mathrm{J})
$$

where $K$ is the total number of SUs, $\rho_{k, n}$ is an integer variable that can be either 1 (if the $n$th subcarrier is occupied by the $k$ th SU) or 0 (otherwise), $\gamma_{k, n}$ is the CINR for the $n$th subcarrier used by the $k$ th $\mathrm{SU}$, and $p_{k, n}$ is the power allocated to the $n$th subcarrier employed by the $k$ th SU. The aforementioned metrics improve the EE of the whole system without guarantee on the achieved EE of individual users. The following worst EE metric improves fairness (in terms of EE), as it maximizes the EE of the user with the lower EE value. It can be defined as [49]

$$
\eta_{\mathrm{EE}, \text { inst }}^{\mathrm{worst}}=\min _{k} \quad w_{k} \frac{B \sum_{n=1}^{N}\left(1-\phi_{\mathrm{md}}\right) \rho_{k, n} \log _{2}\left(1+\gamma_{k, n} p_{k, n}\right)}{\frac{1}{\zeta} \sum_{n=1}^{N} p_{k, n}+p_{\mathrm{c}}} \quad(\mathrm{bits} / \mathrm{J}),
$$

where $w_{k}$ is the relative weight of the EE of the $k$ th SU, being used to reflect certain fairness between SUs and $\phi_{\mathrm{md}}$ is the posterior probability of miss-detection (i.e., when certain PUs bands are identified to be vacant while they are truly occupied). Maximizing $\eta_{\mathrm{EE} \text {,inst }}^{\text {wort }}$ can be viewed as the maximization of the minimum (worst) EE for all SUs to guarantee fairness between users. However, this comes at the expense of deterioration of the average EE of the system. To strike a balance between performance and fairness, the following weighted average EE is additionally defined as

$$
\eta_{\mathrm{EE}, \text { inst }}=\sum_{k=1}^{K} w_{k} \frac{B \sum_{n=1}^{N}\left(1-\phi_{\mathrm{md}}\right) \rho_{k, n} \log _{2}\left(1+\gamma_{k, n} p_{k, n}\right)}{\frac{1}{\zeta} \sum_{n=1}^{N} p_{k, n}+p_{\mathrm{c}}} \quad \text { (bits/J). }
$$


For the single carrier (SC) single-input single-output (SISO) systems, the definition of the instantaneous EE may vary depending on the considered scenario. For example, for a CR base station serving $K$ users in a time synchronized manner on a set of $N$ channels, the instantaneous $E E$ is defined to consider the energy consumed in switching the SU RF chain from a frequency band in a certain frame to another frequency band in the following frame as [10]

$$
\eta_{\mathrm{EE}, \text { inst }}=\frac{\sum_{k=1}^{K} \sum_{n=1}^{N} \rho_{k, n} r_{k, n}\left(t-t_{k, n}^{\mathrm{cs}}\right)}{\sum_{k=1}^{K} \sum_{n=1}^{N} \rho_{k, n} E_{k, n}+\sum_{k=1}^{K}\left(1-\sum_{n=1}^{N} \rho_{k, n}\right) E_{k}^{\mathrm{idle}}} \quad(\mathrm{bits} / \mathrm{J}),
$$

where $r_{k, n}$ is the rate of the $k$ th user employing the $n$th channel, $t$ is the total frame duration, $t_{k, n}^{\mathrm{cs}}$ is the time required by the $k$ th user to tune to the $n$th channel, $E_{k, n}$ is the energy consumed by the $k$ th user to employ the $n$th channel (it is function of transmission duration, channel switching duration, idling duration, transmit power, channel switching power, idling power, and circuitry power), and $E_{k}^{\text {idle }}$ is the energy consumed by the $k$ th user while being in idle state (that is a function of idling power and idling duration). For an SC multiple-input multiple-output (MIMO) broadcast system, the instantaneous EE is defined as [34]

$$
\eta_{\mathrm{EE}, \text { inst }}=\frac{\sum_{k=1}^{K} r_{k}}{\frac{1}{\zeta} \sum_{k=1}^{K} \operatorname{Tr}\left(\mathbf{Q}_{k}\right)+p_{\mathrm{c}}} \quad(\text { bits } / \mathrm{J})
$$

where $r_{k}$ is the rate of the $k$ th $\mathrm{SU}, \mathbf{Q}_{k}$ is the covariance matrix of the $k$ th SU signal, and $\operatorname{Tr}(\mathbf{X})$ denotes the trace of matrix $\mathbf{X}$.

When the instantaneous channel coefficients are not available, an average EE is defined as the ratio of the average transmission rate (or throughput) to the average transmit and consumed circuitry power (or energy) [2, 7, 39-41, 47]. For example, for an SC SISO scenario where an SU overlays a PU and concurrent transmission is required, the average EE is defined as [40]

$$
\eta_{\mathrm{EE}, \mathrm{av}}=\frac{B \log _{2}\left(1+\Delta\left(d_{\mathrm{PS}} / d_{\mathrm{SS}}\right)^{\alpha}\right)}{p_{\mathrm{SU}}} \quad(\text { bits/J), }
$$

where $\Delta$ is the step of the power control, $d_{\mathrm{PS}}$ and $d_{\mathrm{SS}}$ are the distances from the $\mathrm{PU}$ transmitter to the SU receiver and from the SU transmitter to the SU receiver, respectively, $\alpha$ is the path loss exponent, and $p_{\mathrm{SU}}$ is the $\mathrm{SU}$ transmit power. The transmission rate in the numerator of (8) considers the signal-to-interference ratio of the SU link as $p_{\mathrm{SU}}\left(1 / d_{\mathrm{SS}}\right)^{\alpha} / p_{\mathrm{PU}}\left(1 / d_{\mathrm{PS}}\right)^{\alpha}$, where $\Delta=p_{\mathrm{SU}} / p_{\mathrm{PU}}$ and $p_{\mathrm{PU}}$ is the $\mathrm{PU}$ transmit power. For a single node periodic sensing, the average EE can be defined to consider both the time duration and consumed power in the sensing, transmission, and idling as [47]

$$
\eta_{\mathrm{EE}, \mathrm{av}}=\frac{\phi_{\mathrm{PU}}^{\mathrm{idle}}\left(1-(1+w) \phi_{\mathrm{fa}}\right) t^{\mathrm{tr}} r}{\left(p^{\mathrm{se}}-p^{\mathrm{idle}}\right) t^{\mathrm{se}}+T p^{\mathrm{idle}}+\left(p^{\mathrm{tr}}-p^{\mathrm{idle}}\right) t^{\mathrm{tr}}\left(\phi_{\mathrm{PU}}^{\text {busy }} \phi_{\mathrm{md}}+\phi_{\mathrm{PU}}^{\mathrm{idle}}\left(1-\phi_{\mathrm{fa}}\right)\right)}
$$


where $\phi_{\mathrm{PU}}^{\mathrm{idle}}$ and $\phi_{\mathrm{PU}}^{\text {busy }}$ are the probabilities that the PU is not using the channel and occupying the channel, respectively, and $\phi_{\mathrm{fa}}$ and $\phi_{\mathrm{md}}$ are the probabilities of falsealarm and mis-detection, respectively. $t^{\mathrm{se}}$ and $t^{\mathrm{tr}}$ are the sensing and transmission durations, respectively, and $p^{\text {se }}$ and $p^{\text {tr }}$ are the sensing and transmission powers, respectively. $r$ is the transmission rate, the total slot length $T$ (that includes idling, sensing, and transmission durations) and $w$ is a weight that reflects the loss of the potential reward (i.e., number of transmitted bits). The transmission rate in (9) can be expressed as the difference between the transmission reward $\phi_{\mathrm{PU}}^{\text {idle }} t^{\mathrm{tr}} r$ (i.e., when the SU correctly detects the PU's absence, and hence, the SU transmits) and the waste of potential reward $\phi_{\mathrm{PU}}^{\text {idle }}(1+w) \phi_{\mathrm{fa}}{ }^{\text {tr }} r$ (i.e., when the SU falsely reports the PU's presence, and hence, the SU does not transmit). Additionally, the average consumed energy consists of the following parts: 1) the energy when the SU successfully detects the PU's presence, and hence, the SU does not transmit with a probability $\left.\phi_{\mathrm{PU}}^{\text {busy }}\left(1-\phi_{\mathrm{md}}\right), 2\right)$ the energy when the SU mis-detects the PU's existence, and hence, the SU transmits with probability $\left.\phi_{\mathrm{PU}}^{\text {busy }} \phi_{\mathrm{md}}, 3\right)$ the energy when the SU falsely detects the PU's existence, and hence, the SU does not transmit with probability $\phi_{\mathrm{PU}}^{\text {idle }} \phi_{\mathrm{fa}}$ and 4$)$ the energy when the SU correctly the PU's presence, and hence, the SU transmits with probability $\phi_{\mathrm{PU}}^{\mathrm{idle}}\left(1-\phi_{\mathrm{fa}}\right)$. Similarly, the average $\mathrm{EE}$ is defined to consider the SU circuity power and the PU activity during the SU transmission as [41]

$$
\eta_{\mathrm{EE}, \mathrm{av}}=\frac{\phi_{\mathrm{PU}}^{\mathrm{idle}}\left(1-\phi_{\mathrm{fa}}\right) t^{\mathrm{tr}}\left(1-\phi_{\mathrm{PU}}^{\mathrm{re}}\right) r}{\left(p^{\mathrm{se}}+p_{\mathrm{c}}\right) t^{\mathrm{se}}+\left(\frac{1}{\zeta} p^{\mathrm{tr}}+p_{\mathrm{c}}\right) t^{\mathrm{tr}}\left(\phi_{\mathrm{PU}}^{\mathrm{busy}} \phi_{\mathrm{md}}+\phi_{\mathrm{PU}}^{\text {idle }}\left(1-\phi_{\mathrm{fa}}\right)\right)}
$$

where $\phi_{\mathrm{PU}}^{\mathrm{re}}$ is the probability that the PU reoccupies the channel during the SU transmission.

For a single node sequentially sensing $n=1, \ldots, N$ channels, the average EE is defined as [39]

$$
\eta_{\mathrm{EE}, \mathrm{av}}=\frac{\mathbb{E}\left\{\sum_{n=1}^{N} \phi_{n} t^{\mathrm{tr}} r_{n}\right\}}{\mathbb{E}\left\{\sum_{n=1}^{N} E_{n}^{\mathrm{se}}+E_{n}^{\mathrm{tr}}\right\}} \quad(\mathrm{bits} / \mathrm{J})
$$

where $\phi_{n}$ is the posterior probability that the PU channel $n$ is identified to be idle while it is truly idle and $\mathbb{E}$ is the expectation operator. As can be seen, the numerator of (11) accounts for the average number of transmit bits, while the denominator accounts for the total average consumed energy. In a cooperative sensing scheme, the average EE can be defined to account for the total energy consumed by all SUs in the sensing process as well as the energy required for transmission as [2]

$$
\eta_{\mathrm{EE}, \mathrm{av}}=\frac{\phi_{\mathrm{PU}}^{\mathrm{ddle}}\left(1-\phi_{\mathrm{fa}}\right) r t^{\mathrm{tr}}}{E^{\mathrm{se}}+E^{\mathrm{tr}}} \quad(\text { bits } / \mathrm{J})
$$

where $E^{\mathrm{se}}$ and $E^{\mathrm{tr}}$ are the energies consumed by all the cooperative sensing SUs and during the transmission, respectively. 
It is worthy to mention that the inverse of either the instantaneous EE, i.e., $\eta_{\mathrm{EE}, \text { inst }}^{\text {inv }}=1 / \eta_{\mathrm{EE} \text {,inst }}[25]$ or the average EE, i.e., $\eta_{\mathrm{EE}, \mathrm{av}}^{\text {inv }}=1 / \eta_{\mathrm{EE}, \mathrm{av}}[1]$ can be used as a measure of the EE.

\subsection{Energy consumption (EC) metrics}

The total instantaneous/average consumed energy in a given transmission can be also used as an energy-aware metric, and it is measured in joule $(\mathrm{J})[18,24,26,30$, $33,46,48]$.

For example, an energy-aware metric that considers the transmit power in addition to the the power consumed in the analog circuit component, bit resolution of the analog-to-digital converter (ADC), and the input backoff of the power amplifier is expressed as [18]

$$
\eta_{\mathrm{EC}, \text { inst }}=p^{\mathrm{PA}} t^{\mathrm{tr}}+\left(p^{\mathrm{LNA}}+p^{\mathrm{ADC}}\right)\left(t^{\mathrm{tr}}+t^{\mathrm{se}}\right) \quad(\mathrm{J})
$$

where $p^{\mathrm{PA}}, p^{\mathrm{LNA}}$, and $p^{\mathrm{ADC}}$ are the powers consumed in the power amplifier, low noise amplifier, and ADC, respectively.

For a single node sensing, the instantaneous energy consumption of multiple SUs sharing the spectrum with multiple PUs is expressed as [24]

$$
\eta_{\mathrm{EC}, \text { inst }}=\sum_{k=1}^{K} t_{k}^{\mathrm{tr}}\left\|\mathbf{p}_{k}\right\|_{2}^{2} \quad(\mathrm{~J})
$$

where $\mathbf{p}_{k}$ is the transmit beamforming vector of the $k$ th user. The average energy consumption of a two-tier heterogenous network, where the 2nd tier network is equipped with SAP with cognitive capabilities, can be expressed as [46]

$$
\begin{array}{r}
\eta_{\mathrm{EC}, \mathrm{av}}=\frac{\varpi_{\mathrm{s}}}{\varpi_{\mathrm{m}}}\left(p^{\mathrm{syn}}\left(t^{\mathrm{se}}+t^{\mathrm{tr}}\right)+\phi_{\mathrm{UE}} \phi_{\mathrm{s}}\left(p^{\mathrm{se}} t^{\mathrm{se}}+\phi_{\mathrm{md}} p^{\mathrm{proc}} t^{\mathrm{tr}}\right)\right. \\
\left.+\left(1-\phi_{\mathrm{UE}}\right) \phi_{\mathrm{s}}\left(p^{\mathrm{se}} t^{\mathrm{se}}+\phi_{\mathrm{fa}} p^{\mathrm{proc}} t^{\mathrm{tr}}\right)\right)
\end{array}
$$

where $\varpi_{\mathrm{m}}$ and $\varpi_{\mathrm{s}}$ are the densities of mobile base station of the 1 st tier network and the small cell access points of the 2 nd tier network, respectively, $p^{\text {syn }}$ and $p^{\text {proc }}$ are the synchronization and processing powers, respectively, $\phi_{\mathrm{UE}}$ is the probability that there is a user equipment within the coverage of an SAP unit, and $\phi_{\mathrm{s}}$ is the success probability of a typical user within the coverage area of SAP.

For a cooperative sensing distributed CR sensor network, where the SU may turn off its sensing and transmission capabilities in order to save energy, the average energy consumption is expressed as [33]

$$
\eta_{\mathrm{EC}, \mathrm{av}}=(1-\mu)\left(\sum_{k=1}^{K} E_{k}^{\mathrm{se}}+E_{k}^{\mathrm{tr}}(1-v)\right) \quad(\mathrm{J})
$$


where $\mu$ is the $\mathrm{SU}$ sleep rate, i.e., the probability that the $\mathrm{SU}$ is turned off and $v$ is the censoring rate, i.e., the probability that the $\mathrm{SU}$ has no output from the sensing process. The average energy consumption of another cooperative spectrum sensing scheme where SUs can be considered as relays can be defined as [30]

$$
\eta_{\mathrm{EC}, \text { av }}=K^{\text {relay }} N_{\text {samples }} T_{\text {samples }} p^{\text {relay }} \quad(\mathrm{J}),
$$

where $K^{\text {relay }}$ is the number of relays, $N_{\text {samples }}$ is the total number of samples, $T_{\text {samples }}$ is the sampling period, and $p^{\text {relay }}$ is the average transmit power of a given relay.

\subsection{Utility metrics}

Utility functions can be used to measure the EE. For example, a utility function that considers the subcarrier availability, and hence, the reliability of transmission is defined as the difference between the SU transmission rate and its prospective rate loss due to sensing errors or to the PU reoccupying the channel during the $\mathrm{SU}$ transmission [27]

$$
U_{\text {rate-loss }}=B \sum_{n=1}^{N} \log _{2}\left(1+\gamma_{n} p_{n}\right)-\phi_{n} \mathscr{L}\left(p_{n}\right) \quad(\text { bits/sec }),
$$

where $\mathscr{L}\left(p_{n}\right)$ is a real-valued increasing concave and normalized average loss function of the power consumed in subcarrier $n$ and $\phi_{n}$ denotes the probability that a PU reoccupies channel $n$ during the SU transmission or the probability that sensing errors occur during the PU transmission. Hence, $\phi_{n} \mathscr{L}\left(p_{n}\right)$ represents the average rate loss due to sensing errors or collision with PU transmission.

Another utility function that uses concepts of multi-objective optimization to maximize the transmission rate with the least amount of transmit power is defined as $[11,22]$

$$
U_{\text {rate-power }}=w_{1} B \sum_{k=1}^{K} \sum_{m=1}^{M} \log _{2}\left(1+\gamma_{k, m} p_{k, m}\right)-w_{2} \sum_{\substack{k=1 \\(\text { dimensionless }),}}^{M}\left\|\mathbf{p}_{k, m}\right\|_{\mathrm{F}}^{2}
$$

where $w_{1}$ and $w_{2}$ are the relative weighting coefficients associated with the competing objectives, $m=1, \ldots, M$ denotes the index of the transmit stream, and $\|\cdot\|_{\mathrm{F}}^{2}$ is the Frobenius matrix norm. Here, the weighting coefficients $w_{1}$ and $w_{2}$ include normalization factors such that the competing objectives are within the same range, and hence, the metric in (19) is dimensionless [11]. It is worthy to mention that if the circuity power is considered in this metric, the optimal EE solution can be achieved at certain values of $w_{1}$ and $w_{2}$ [4]. Similarly, a utility function that maximizes the transmission rate with the least amount of consumed energy in the sensing process is defined as [42] 


$$
\begin{gathered}
U_{\text {rate-energy }}=w_{1} \sum_{n=1}^{N} r_{n}\left(1-\phi_{n, \mathrm{fa}}\right) \phi_{n}^{\text {idle }}-w_{2}\left(t^{\mathrm{se}} p^{\mathrm{se}} K^{\mathrm{se}}+t^{\mathrm{se}} p^{\mathrm{idle}}\left(K-K^{\mathrm{se}}\right)\right) \\
\text { (dimensionless), }
\end{gathered}
$$

where $\phi_{n, \text { fa }}$ is the probability of false alarm of channel $n, \phi_{n}^{\text {idle }}$ is the probability that channel $n$ is idle, and $K^{\text {se }}$ is the number of sensing users.

\subsection{Other metrics}

Other additional dimensionless energy-aware metrics can be used, which capture other aspects of the energy consumed in the transmission. A novel dimensionless energy-aware metric is defined that captures the actual (and total) energy consumed in transmitting (and receiving) one packet, average PU interference time, average PU transmission time, and reliability of transmission as [38]

$$
U_{\text {energy-reliability }}=\frac{p^{\operatorname{tr} \ell}}{p^{\operatorname{tr} \ell}+E_{\mathrm{c}} r}(1-\Lambda)^{\ell} \quad \text { (dimensionless), }
$$

where $\ell$ is the packet length in bits, $E_{\mathrm{c}}$ is the consumed energy of a node before the actual transmission occurs, and $\Lambda$ is the bit error rate. As can be seen, $p^{\text {tr }} \ell$ represents the actual energy required to transmit one packet, $p^{\mathrm{tr}} \ell+E_{\mathrm{c}}$ is the total energy spent to transmit and receive one packet, and $(1-\Lambda)^{\ell}$ accounts for the reliability of transmission, i.e., all the $\ell$ bits are received correctly.

A novel metric that captures the sensing gain of cooperative spectrum sensing is defined as the cooperative sensing gain in $\mathrm{dB}$ per joule per SU [29]

$$
\eta_{\text {gain }}=\frac{\beta}{K^{\text {se }} E^{\text {se }}} \quad(\mathrm{dB} / \mathrm{J} \text { per } \mathrm{SU}),
$$

where $\beta$ is the cooperative sensing gain in $\mathrm{dB}$ and it is defined as the difference between the mis-detection threshold and the probability of mis-detection of cooperative spectrum sensing.

\section{Energy-Aware Resource Allocation}

In order to improve the energy efficiency performance of CR systems, different parameters contributing to the total energy consumption can be optimized, e.g., transmit power, power consumed in the transmitter and receiver circuitry, allocation of subchannels, sensing duration, idle duration, transmission duration, sensing access strategy, number of sensing SUs, and/or SUs assignment to sense the channel $[1,2,7,10,12,18,22,24-27,29,30,33-35,38-42,44-49]$. When the energy consumed in the sensing process can be neglected, then transmit power, 


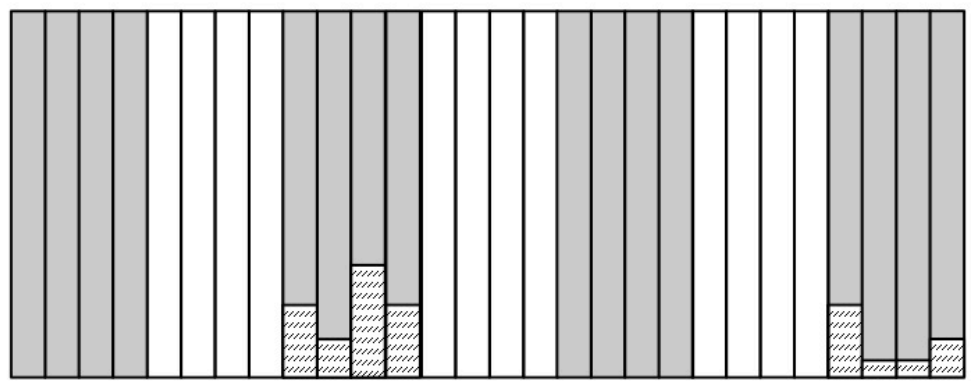

subcarriers PU band

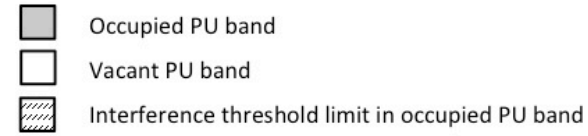

Fig. 2 Side-by-side frequency spectrum model.

consumed circuitry power, and/or frequency bands/subcarriers are optimized to improve the energy efficiency performance of the CR networks $[1,10,12,22,25$, $27,34,35,38,40,44,45,49,51]$. Otherwise, additional parameters have to be optimized such as sensing duration, idling duration, transmission duration, sensing access strategy, number of sensing SUs, and/or SUs assignment to sense the channel $[2,7,18,24,26,29,30,33,39,41,42,46-48]$, in addition to adjusting the previous parameters accordingly.

\subsection{Sensing-less energy-aware resource allocation}

Energy-aware CR systems can be designed while neglecting the effect of the energy spent in the sensing process. In such a case, transmit power [1, 27, 34, 35, 40, 45], signal-to-interference-plus-noise ratio (SINR) threshold [22], frequency bands/subcarriers [9, 25, 44, 49], and/or packet length [38] are optimized separately or jointly in order to improve the CR system energy efficiency.

\subsubsection{Power allocation of multicarrier systems:}

OFDM is recognized as an attractive modulation technique for CR networks due to its spectrum shaping flexibility, adaptivity in allocating vacant radio resources, and capability in monitoring the spectral activities of PUs [32]. The available spectrum can be accessed by a single SU employing OFDM modulation [27, 34, 45] or by multiple SUs employing OFDMA [25, 44, 49]. In case of a single SU accessing the spectrum, the power allocated to each OFDM subcarrier should be optimally allocated in order to improve EE. On the other hand, if multiple SUs access the spec- 
trum, the subcarriers assigned to different SUs should be additionally optimized. In either case, a side-by-side frequency spectrum model is adopted as shown in Fig. 2, where the available frequency spectrum is divided and licensed to multiple PUs that do not necessarily use it all the time or at all the geographical locations [8].

In a simple overlay coexistence scenario, one SU is assumed to coexist adjacent in the frequency domain with one PU [45]. To further simplify the scenario, perfect CSI is assumed to be known between the SU transmitter and receiver pair, between the SU transmitter and the PU receiver, and between the PU transmitter and the SU receiver. This assumption is practically challenging; however, CSI can be obtained through cooperation/feedback between the PUs and SUs with negligible error, especially at high SNR [52]. The CR system uses the instantaneous knowledge of the CSI and optimizes the transmit power in order to maximize $\eta_{\mathrm{EE} \text {,inst }}$ in (1) of the SU while guaranteeing total power constraint to reflect the SU power amplifier limitations and adjacent interference constraint to control the amount of the leaked interference to the PU. The formulated optimization problem is non-convex and it is solved using concepts of fractional programming, i.e, Dinkelbach method [21]. Results show that the EE can be maximized at the expense of deteriorating the SU rate.

Towards a more practical scenario where the SU coexists with multiple PUs in the overlay approach and guarantees certain QoS requirements, a minimum supported rate is added to the optimization problem [35]. A novel method, namely the generalized waterfilling factor aided search (WFAS), is proposed to solve the nonconvex optimization problem [35]. A simplified version of the WFAS method is further presented. This version has simpler structure when compared to the Dinkelbach method in [45], i.e., the EE optimization problem is solved through the well-known rate maximization problem. Hence, the complexity order of the proposed simplified WFAS is much lower than its counterpart in [45].

A more practical scenario of an SU coexisting with multiple PUs is when the SU does not rely on perfect CSI knowledge [12]. Channel estimation errors on the links between the SU transmitter and receiver pair are considered. Additionally, the SU does not have perfect sensing capabilities and only knows the statistics of the channels to the PUs receiver. The formulated optimization problem minimizes the SU instantaneous EE (the inverse of (2)) while guaranteeing certain power budget, minimum supported QoS, limited co-channel interference (CCI), and limited adjacent channel interference (ACI) constraints. The non-convex optimization problem is transformed to an equivalent one and solved using Dinkelbach algorithm [21]. Numerical results show that increasing the channel estimation errors deteriorates the EE. Additionally, the assumption that the SU has perfect sensing capabilities leads to violation of the interference constraints in practice as can be seen in Fig. 3. In other words, if the SU is assumed to sense the PUs activities perfectly, which is not necessarily true in practical scenarios, then the interference received at the PUs can exceed the predefined threshold. Hence, the practical case of the SU with limited sensing capabilities should be considered.

A generalization to the previous scenarios is when the SU accesses the spectrum in both overlay and underlay approaches [27]. In such a scenario, the SU is allowed 


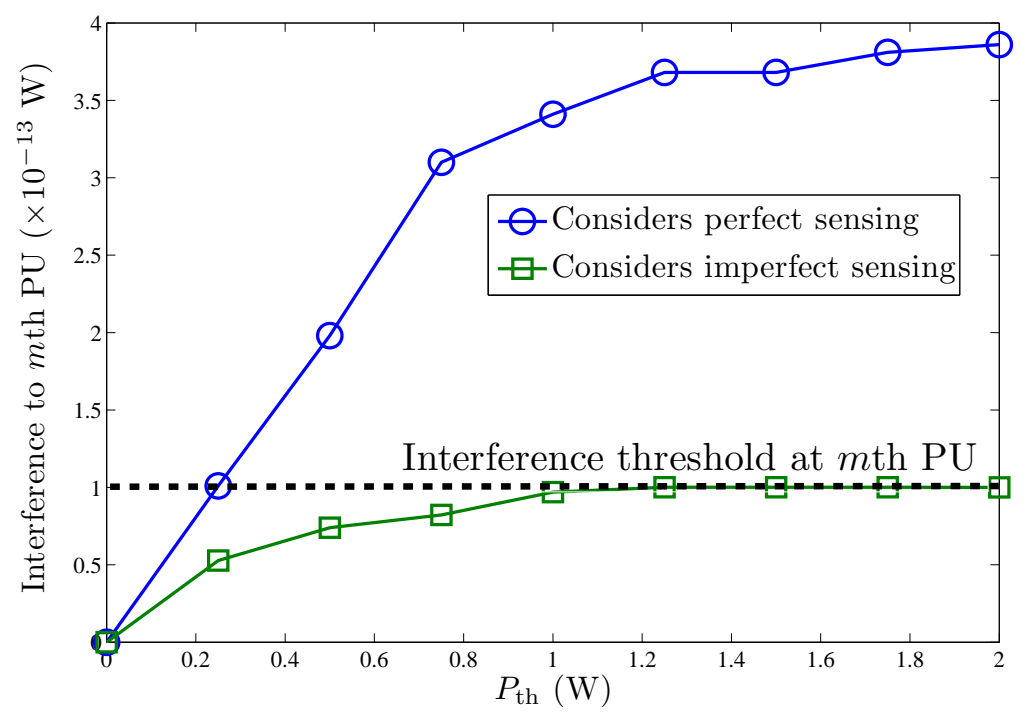

Fig. 3 Effect of perfect and imperfect sensing assumptions on the interference leaked to the $m$ th PU. Simulation parameters are as in [12].

to use the frequency band of a PU (underlay) that is located at a distant geographical location from the PU, and hence, it is required to transmit with lower transmit power such that no harmful interference occurs to the co-channel PU. This is in addition to the SU coexistence with PUs in unused adjacent frequency band (overlay). Due to practical considerations, only knowledge of the distance-based path loss is taken into account on the links between the SU transmitter and the PUs receivers. The SU considers a risk-return model to maximize $U_{\text {rate-loss }}(18)$, where the power allocated to a certain subcarrier is recognized as an investment, while loss of useful power when the PU reoccupies the band is recognized as a risk. The optimization problem additionally imposes total transmit power, CCI, and ACI constraints. Optimal and suboptimal algorithms are proposed to show the performance of the SU. It was shown that valuable resources can be saved, e.g., battery life, by selectively allocating higher power to underutilized subcarriers and lower power to subcarriers with high PUs activities.

\subsubsection{Power and subcarrier allocation of multicarrier systems:}

When multiple SUs are allowed to access the spectrum of multiple PUs [25, 44, 49], the aforementioned models can be extended as follows. An underlay downlink scenario is considered where a cognitive base station and multiple SUs receivers share the licensed spectrum of PUs [44]. The SUs access technology is assumed to be 
OFDMA and perfect CSI is assumed between the base station and the SU receivers, between the CR base station and the PUs receivers, and between the PUs transmitters and the SUs receivers. The CR base station allocates the power and subcarriers of the SUs in order to maximize $\eta_{\mathrm{EE} \text {,inst }}$ in (3) of the whole CR network subject to total transmit power, ACI, and certain QoS of each SU (in terms of minimum supported rate) constraints. The formulated problem is a mixed-integer programming problem that is computationally hard to solve. In order to overcome the complexity burden, the concept of time-sharing is adopted, i.e., two different SUs can share the same subcarrier, and then a hypograph form is used to convert the resultant nonconvex optimization problem into a convex one, where a barrier method [16] is used to find the solution.

Another possible scenario is when the available OFDMA resources (i.e., power and subcarriers) are optimally distributed to enable communications between the SU transmitter and receiver pairs [25]. An optimization problem is formulated for each SU transmitter and receiver pair in order to minimize $\eta_{\mathrm{EE}, \text { inst }}^{\text {inv }}$ (the inverse of (3)) of each pair, while guaranteeing acceptable interference to existing users (PUs and SUs with already established connections) and minimum supported rate for each SU pair. The proposed solution is named energy-efficient waterfilling solution, where the optimality is obtained in the constraint interval. This is in contrast to the classical waterfilling solutions to maximize the rate or margin, where the optimality point is found at the constraint boundary. Numerical results show that for multiple transmitter and receiver pairs employing OFDMA, the optimal EE solution for a certain transmitter and receiver pair does not necessarily select the best subcarrier for transmission. This is in contrast to spectral efficiency maximization problems, where the optimal solution for a given pair selects the best subcarrier for transmission.

An upper bound on the performance is achieved when perfect CSI is assumed $[25,34,44,45]$. In practical scenarios where perfect CSI is not available, the achieved performance is expected to degrade. In fact, only knowledge of average channel power gain on the links between the SUs transmitters to the PUs receivers is assumed in [49]. The worst $\eta_{\mathrm{EE}, \text { av }}^{\text {worst }}$ and average $\eta_{\mathrm{EE}, \mathrm{av}} \mathrm{EE}$ metrics in (4) and (5), respectively, are to be optimized while guaranteeing a predefined fairness between SUs. In order to maintain fairness between users, the lowest SU EE is maximized and in order to improve the EE of the CR network as a whole, the summation of the weighted SUs EE is maximized, i.e., average EE. The formulated optimization problems are non-convex integer optimization problems that guarantee total transmit power and per subcarrier average interference ${ }^{2}$ constraints and neglect ACI constraint. The optimization problems are relaxed to convex ones, where a general concave envelope function is used to find a near-optimal solution.

\footnotetext{
${ }^{2}$ This is to guarantee PU protection in case of incorrect sensing of the channel.
} 


\subsubsection{Power allocation of single carrier systems:}

Other systems that adapt the power may not employ OFDM(A) to access the available spectrum in CR networks $[1,22,35,40]$. In such cases, single carrier for SISO systems [40] or MIMO systems [1, 22, 35] can be used. For example, an interference-limited spectrum sharing CR ad-hoc network is considered in [40], where simultaneous transmission between an SU pair and a PU pair is requested. A forbidden transmission region around the $\mathrm{PU}$ receiver is defined, where no $\mathrm{SU}$ transmission is allowed to guarantee the PU QoS. On the other hand, an effective cognitive region is defined, where the SU transmission is allowed if and only if the QoS of the SU can be achieved (in this case, a concurrent transmission between both the PU pair and the SU pair is possible while meeting both users QoS). The step size of the SU power control is adapted in order to maximize $\eta_{\mathrm{EE}, \mathrm{av}}$ in (8) while neglecting the SU circuitry power consumption. It was shown through simulation examples that the concurrent transmission region can be expanded if power control is used.

Multiple SUs share the spectrum in an underlay fashion with a single PU in [22]. It is assumed that the SUs and the PU have MIMO capabilities. The target is to maximize the transmission rate and the number of admitted SUs to use the spectrum with the least possible transmit power. Accordingly, a MOOP problem is formulated to maximize $U_{\text {rate-power }}(19)$ subject to peak power and interference constraints. The formulated problem considers the effect of imperfect CSI (including quantization and estimation errors) through adopting a normal-bounded channel imperfection model [43]. In addition to the power, the SINR threshold is considered as one of the optimization variables in order to have an efficient admission control without additional integer variables. In other words, a certain data stream of a certain SU is admitted for transmission if its received SINR is above a certain optimal threshold. The MOOP problem utility function is combined into a single objective function using the weighting sum method, where weighting coefficients are adopted to reflect the relative importance of the competing rate and power. The resulting problem is solved using concepts of semidefinite programming [16].

A MIMO boradcast scenario is considered in [34], where a single SU transmitter and multiple SUs receivers are assumed to share the spectrum in an underlay fashion with multiple PUs [34]. To maximize $\eta_{\mathrm{EE} \text {,inst }}$ in (7), an optimization problem is formulated subject to transmit power, interference, and minimum supported rate constraints. The problem is non-convex and in order to reach the optimal solution, it was transformed into a one-dimensional optimization problem with a quasi-concave objective function that is solved using a golden search. Numerical results show that the EE can be significantly improved at high SNR regime or for a large number of SUs.

An uplink EE scheduling in CR networks is when an SU base station is serving a number of SUs in a time-slotted manner [10]. At the beginning of each frame, each SU gets a list of all the available frequency bands and estimates its capacity to each idle band. The SUs send this information to the base station along with the number of bits in its buffer. The CR base station assigns/schedules one SU to one frequency band in order to maximize $\eta_{\mathrm{EE} \text {,inst }}$ in (6) of all SUs. The CR base sta- 
tion considers the energy consumed in switching the SU RF chain from a frequency band in a certain frame to another frequency band in the following frame. The formulated scheduling optimization problem is solved by the Charnes-Cooper method [6] with high computational complexity. Then, two suboptimal solutions are provided to reduce the complexity. Results indicate that if there are many frequency bands to schedule, then a significant improvement in the EE is achieved when compared to other works in the literature that maximize the capacity. On the other hand, if there is a limited number of frequency bands, then the achieved EE is comparable to other works that maximize the capacity.

\subsubsection{Packet length allocation:}

For a CR sensor network, increasing the packet size increases the network utilization; however, this is at the cost of increasing the packet loss probability if the PUs reoccupy the channel. On the other hand, reducing the packet size reduces the interference to PUs; however, this suffers from extensive overhead. Accordingly, it is important to find the optimal packet length that maximizes the EE of CR sensor networks. An overlay CR sensor network is considered to operate in sleep or active mode [38]. The sleep mode consists of ready, monitor, observe, and deep sleep periods, while the active mode consists of periods of sensing, decision, handoff, transmit, and receive. An optimization problem to maximize the $U_{\text {energy-reliability }}$ in (21) is formulated subject to interference and maximum distortion level for PU reliable detection constraints, and it is solved using sequential quadratic programming [14]. Results show that changes in the PU behavior in the target BER can significantly change the optimal packet length.

\subsubsection{Relays placement:}

To enhance the EE of CR networks, a new architecture is introduced, namely, cognitive capacity harvesting networks [51]. In such an architecture, SUs are not equipped with cognitive capabilities in order to reduce their energy consumption, and the sensing capabilities are moved to a set of relay stations. The optimal placement of the relay stations significantly affects the EE of the CR network. An optimization problem is formulated to minimize the placement size subject to EE and spectrum efficiency constraints. Due to the NP-hardness of the problem, a two step heuristic algorithm is proposed to find a near-optimal solution. Results show that the proposed heuristic algorithm outperforms the random placement and the number of required relay stations are at most twice the number obtained from the optimal solution. 


\subsection{Sensing-aware energy-aware resource allocation}

The sensing process plays an important role in EE optimization in CR networks $[7,18,24,26,29,30,33,39,41,42,46,47]$. Increasing the sensing time improves the detection probability at the expense of consuming more energy, whereas reducing the sensing time results in more collisions with existing PUs due to false detection. Furthermore, increasing the transmission time increases the CR network performance; however, data loss may happen as PUs may reoccupy the channel at any point in the SU transmission. For a fixed frame duration, reducing the transmission time directly limits the network performance and leads to longer sensing duration, and hence, wastes energy. A single SU can sense the available spectrum for possible opportunities for its transmission [18, 24, 26, 39, 41, 46-48] or multiple SUs can perform the sensing in a cooperative manner in order to improve the sensing performance at the expense of spending more energy [7, 29, 30, 33, 42].

\subsubsection{Single node sensing}

\subsubsection{Periodic sensing:}

For a single SU sensing the channel, a general time-slotted CR system is considered where the frame is divided between sensing, transmission, and possible idling ${ }^{3}$ durations [47]. The sensing and transmission durations are optimized to maximize $\eta_{\mathrm{EE}, \mathrm{av}}$ in (9) subject to the probability of detection and maximum energy consumption constraints. Results show that shorter sensing durations can be achieved at the expense of increasing the sensing power, while larger idling power requires longer sensing durations. A more practical scenario is encountered when the PUs can reoccupy the channel during the SU transmission [41]. Stringent QoS of the SU is assumed, and hence, the successful transmission of the SU occurs if and only if the whole frame is sent correctly. Consequently, the sensing and transmission durations are optimized in order to maximize $\eta_{\mathrm{EE} \text {,inst }}$ in (10) subject to detection probability and interference to PUs constraints. The optimization problem is non-convex, and a sub-optimal alternate search algorithm is proposed to reach an acceptable solution. It was shown through numerical evaluations that significant improvements in the EE can be achieved by optimizing the sensing/transmission duration when compared to fixed sensing/transmission duration techniques [31].

Another scenario of single node sensing is discussed for heterogenous networks (HetNets) to improve their EE [46]. In HetNets, macro-cells are deployed to guarantee coverage, being overlayed by small cells (i.e., micro-cells, pico-cells or femtocells) that offload traffic from the macro-cells in order to support local traffic demands [5]. However, deployment of small cells base stations is accompanied by excessive energy consumption [5]. Traffic demands may fluctuate over time, frequency, and space; hence, sleep mode techniques can be used to reduce the en-

${ }^{3}$ Idle duration is important when the SU has to stop its transmission when a PU reoccupies the channel. 
ergy consumption [19]. To reduce the signalling overhead and hence improve the $\mathrm{EE}$, a distributed sleep mode that does not require user location information is proposed in [46]. This is achieved by adopting small cell access points (SAPs) that have cognitive capabilities to periodically sense the channel if a user is active within its coverage area. The sensing time and probability are optimized to minimize $\eta_{\mathrm{EC}, \mathrm{av}}$ in (15) in synchronization, sensing, processing, and transmission, while considering random locations of the users within the SAP coverage area. Results showed the tradeoff between the energy consumption and the amount of traffic that can be offloaded from macro-cells and it was shown that the consumed energy linearly increases with the density of the SAPs. Additionally, it was shown that knowledge of the interference environment can lead to significant reduction of the SAPs energy consumption.

The energy consumption of a CR sensor network can be reduced by selecting the operation mode, i.e., channel sensing, channel switching, and data transmission [26]. The CR sensor network is assumed to sense the channel continuously in order to identify vacant PU bands. In order to guarantee the PU protection, SUs need to switch the channel as fast as possible if a PU returns to use the channel. To reduce the switching time, the CR sensor network prepares a candidate channel called backup channel. An algorithm that considers errors in the sensing process is proposed to select the operation mode using the concepts from the partially observable Markov decision process [36].

In an underlay approach, multiple SUs are assumed to share the spectrum with multiple PUs [24] and both the SUs and PUs can be equipped with single or multiple antennas to access the spectrum. An uplink scenario is considered where the SUs transmitters communicate with a single SU base station using the time division multiple access protocol. The sensing time and the beamforming of the SUs is optimized in order to minimize $\eta_{\mathrm{EC} \text {,inst }}$ in (14) for the SUs while satisfying transmit power, interference to PUs, and QoS (in terms of minimum supported rate) for the SUs constraints. The SU is assumed to have either perfect or imperfect CSI on the links to the PUs receivers. In case of the availability of perfect CSI, the interference constraints have to be satisfied in a deterministic way, i.e., for every channel realization. In such case, closed form expression for the optimal solution can be reached if the SUs are under-utilized and a heuristic sub-optimal solution is proposed if the SUs are heavily-utilized. On the other hand, if perfect CSI is not available, the interference constraint has to be satisfied in a statistical way and the optimization problem is solved through decomposition [16]. Numerical results show that the energy consumption is significantly reduced compared to works that maximize the transmission rate, with less generated interference to PUs.

Another perspective of minimizing the energy consumption in delay-constrained CR systems is achieved when the SU adapts its transmission rate, i.e., reduces its transmission rate under deep fading channel and increases the rate for good channel conditions, in order to deliver a target payload [48]. The optimization problem is formulated as a discrete-time Markov decision process [36], where a low complexity algorithm is proposed to find the optimal policy. The results show that the impact of 
energy overheads is more significant for delay-insensitive traffic when compared to delay-sensitive scenarios.

For short range communications, the energy consumption in the transmitter and receiver circuitry is more significant than the energy required for transmitting the data [20]. Therefore, optimizing the sensing/transmission duration only is not sufficient and other parameters affecting the energy consumed in the circuitry should be optimized as well [18]. The optimization variables are the sensing duration, the input backoff of the power amplifier, the power consumed in the low noise amplifier, and the bit resolution of the ADC. During the sensing duration, only the energy consumed in the SU receiver is considered, i.e., the energy consumed in the low noise amplifier and ADC. On the other hand, during the transmission duration, the power consumed in the power amplifier of the SU transmitter is additionally considered [18]. The results show that for strong interference from the PU, less transmission energy and more circuit energy are required. Additionally, the SU receiver, especially ADC, consumes more energy when compared to traditional systems, and hence, it is preferable to operate the power amplifier of the SU transmitter at high input backoff values to compensate for such energy loss.

\subsubsection{Sequential sensing:}

Unlike periodic sensing [18, 24, 26, 41, 46-48], a sequential sensing approach is optimized in order to maximize the average EE [39]. In sequential sensing, the SU has to make a decision on whether to continue to sense the next channel or to start its transmission, i.e., a sequence of decisions has to be made before the SU transmission. Sequential sensing is efficient as it allows the SU to sense another channel if the the current sensed channel is identified to be busy, instead of waiting until the beginning of the next frame. The sensing-access strategies to be optimized are the sensing strategy (i.e., when to stop sensing and start transmission), access strategy (i.e, determine the transmit power level during the transmission), and the sensing order (i.e, which channel to sense if the current channel is changed). The formulated optimization problem is a stochastic sequential decision-making problem, i.e., the decision maker has to decide at each state after observing the current system state [13], that is difficult to solve directly. A sub-optimal solution can be reached by transforming the problem into a parametric formulation that rewards the throughput and penalizes the transmit power. Numerical results show that the sensing strategy has a certain threshold, i.e., the SU transmits if the channel is good enough; otherwise, the SU should keep sensing. Additionally, the optimal power allocation has a waterfilling structure and the optimal sensing order is to choose to sense a channel associated with the maximum expected future net reward, i.e., average EE.

\subsubsection{Cooperative sensing}

Using a single SU to sense the spectrum has its own difficulties and limitations [18, 24, 26, 39, 41, 46-48], e.g., multi-path fading, shadowing, and hidden termi- 


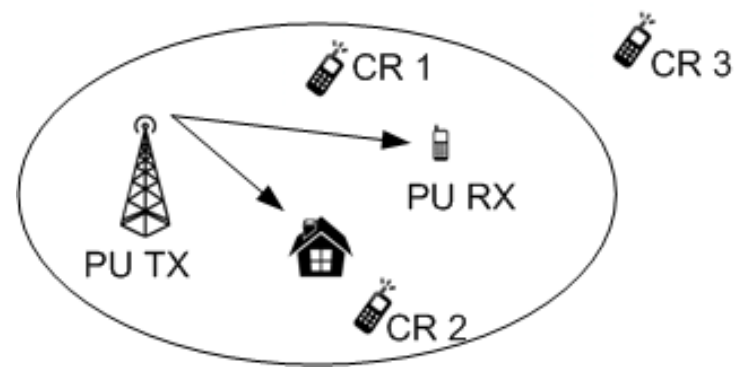

Fig. 4 Cooperative sensing.

nal problem [50]. For example, consider Fig. 4 where CR 2 cannot receive the PU transmission due to shadowing and CR 3 is located outside the PU transmission coverage, and hence, not aware of its transmission. One possible way to overcome these shortcomings is to use cooperative spectrum sensing, where more than one $\mathrm{SU}$ is allowed to collaboratively sense the channel [50]. For example, in Fig. 4 CR 1 can sense the PU signal and share the sensing results with CR 2 and CR 3. One of the main challenges of EE optimization in the cooperative sensing CR environment, when compared to single SU sensing strategies, is to find the optimal number and the assignment policy of SUs participating in the sensing process [7, 29, 30, 33, 42].

\subsubsection{General scheme:}

A general cooperative sensing model can be considered as a two step process, where the first step is to find the number and assignment of SUs to do the sensing and the second step is to determine the sensing duration, detection threshold, etc. The most challenging part of cooperative sensing is related to the first step [29, 42]. The more SUs participating in the sensing process, the higher the sensing accuracy. However, this will be accompanied by an increase in the consumed energy in the sensing process. Some SUs are better candidates for sensing if they are subject to less noise or not in deep fading, and it is assumed that each SU can only be assigned to sense one frequency band in a given time slot [29]. In this case, the optimization problem reduces to finding the optimal number of SUs to sense the spectrum and to find which SU is assigned to sense a certain channel. A greedy algorithm is used to find the optimal solution and it was shown that significant performance gain is achieved especially with a large number of SUs [29].

\subsubsection{Sensing and relaying scheme:}

Another possible scenario for cooperative spectrum sensing is when SUs can be considered as relays as well [30]. In this case and during a first phase, all relays monitor the activity of a single PU and in a second phase, all relays amplify their PU received signal and send it to a data fusion center. In such a scenario, the optimal number of sensing/relays SUs, their amplifying gain, and the number of processed 
samples are to be optimized in order to reduce the energy consumption subject to the probabilities of false-alarm and detection constraints. In modeling the energy consumption, the circuit energy consumption in the relays, in the fusion center, and in the PUs are neglected under the condition that the transmission distance from the PU to the relays and from the relays to the fusion center is large. The formulated optimization problem is solved using an exhaustive search which is computationally complex. In order to reduce the complexity, equal power allocation of the relays is assumed and closed-form expressions for the optimal number of samples and the number of relays were obtained. It was shown that for higher PU transmit power, a reduced number of relays or samples is needed [30].

\subsubsection{Sleep-mode based cooperative scheme:}

Another scenario for distributed spectrum sensing is considered in [33], where the target is to minimize $\eta_{\mathrm{EC} \text {,av }}$ in (16) subject to a minimum probability of detection and a maximum false alarm probability constraints. In order to achieve this target, a sleep mode rate and censoring thresholds are to be optimized. In the sleep-mode, an SU turns off its sensing and transmission functions in order to save energy. On the other hand, censoring thresholds define the detection thresholds where an SU is confident of its sensing results. This can be explained as follows, if the output of the energy detector is greater than a certain threshold, then the SU reports its sensing decision as the PU exists; if the output of the energy detector is less than a certain threshold, then the SU reports its sensing decision as the PU does not exist; and if the output of the energy detector is between these two thresholds, the SU makes no decision and enters the sleep-mode. The optimization problem is solved using the alternating search algorithm [16] under two setups: the blind setup, when there is no prior information about the PU presence and the information-aided setup, when such information is available. The results indicate that when the energy consumed in transmission exceeds the energy consumed in sensing the sleep rate is higher than its counterpart when the the transmission and sensing energies are equal. Additionally, the minimized energy consumption is reduced significantly and becomes almost independent of the number of sensing users for a large number of users.

To conclude the sensing-aware energy-aware resource allocation section, different sensing parameters directly affect EE of CR systems and should be carefully selected. For single node sensing time-slotted systems, the sensing duration plays a crucial rule in maximizing the EE of the system. For instance, increasing the sensing time improves the detection probability at the expense of consuming additional energy, while decreasing the sensing time reduces the energy wasted in the sensing process but at the expense of more collisions with PUs. For cooperative sensing, the problem is even more challenging, as the system designer needs to select the optimal number of SUs to sense the PUs spectrum. For the short range communications when the energy consumption in the circuitry is more than the transmission energy, optimizing the sensing duration is not enough and optimizing the input backoff of the power amplifier becomes crucial. 


\section{Challenges and Opportunities}

The performance of cooperative spectrum sensing can be severely degraded by misbehaving SUs, i.e., malicious users, that provide false information about sensing to the fusion center in order to have a wrong final decision and then use the spectrum exclusively [17]. This is achieved by reporting that the spectrum is occupied such that the fusion center produces a final decision that the spectrum is occupied and no legitimate SUs should access the spectrum; then, the malicious SUs become the only users to access the spectrum. A possible solution to this problem is that the fusion center accepts only reported results from authenticated SUs. This authentication can be achieved by transmitting extra bits for cryptographic purposes; however, additional energy has to be spent in these overhead bits. Then, the number of the security bits has to be optimized in order to maximize $\eta_{\mathrm{EE}, \mathrm{av}}$ in (12) [2]. An average EE metric that captures the influence of malicious users is formulated, i.e., the malicious users tend to increase the false alarm probability, which decreases the successful amount of bits transmitted, and hence, reduces the EE. The optimal number of security bits depends on the fusion rate, fusion rule, number of malicious users, and number of legitimate users. The optimal number of bits increases as the number of malicious users increases, which decreases the EE.

Improving the SU connectivity comes mostly at a cost of increasing the energy consumption in CR systems. One of the most recent approaches to boost the SU performance is the use of improper Gaussian signaling, which creates more opportunity than the traditional proper signaling schemes, to minimize its outage while satisfying PU QoS [3]. Thus, investigating the EE-SE trade-off optimization problem in the underlying SU system is important in order to tune the SU power and circularity coefficient to accomplish an acceptable balance between EE and SE.

Additionally, employing full duplex in CR is considered an efficient way to improve the SE after the recent progress in self-interference cancelation. On the other hand, this may not be the case as discussed in a generic MIMO wireless communication system [37]. Since the CR has additional channel sensing cost, designing energy-efficient full-duplex CR system is a challenging issue. To tackle this issue, CRs need to operate with new adaptive full duplex modes and polices to accomplish a balance between efficient sensing, SE, and EE.

Another promising trend to improve the EE of future CR networks is through energy harvesting CRs This is achieved by tapping energy from readily available ambient sources, e.g., wind, solar, and radio frequency signals. However, knowing the instantaneous energy arrival is crucial in order to optimize the performance of energy harvesting CR networks. Energy arrivals can be assumed to be available in order to simplify the optimization framework. However, such an assumption may not be realistic; in this case, statistical knowledge about the energy arrival can be assumed. It is expected that energy harvesting will extend the lifetime of CR networks with improved EE performance. 


\section{References}

[1] Akin S, Gursoy MC (Sep. 2013) On the throughput and energy efficiency of cognitive MIMO transmissions. IEEE Trans Wireless Commun 62(7):32453260

[2] Althunibat S, Sucasas V, Marques H, Rodriguez J, Tafazolli R, Granelli F (Aug. 2013) On the trade-off between security and energy efficiency in cooperative spectrum sensing for cognitive radio. IEEE Commun Lett 17(8):15641567

[3] Amin O, Abediseid W, Alouini MS (Jun. 2015) Outage performance of cognitive radio systems with improper gaussain signaling. In: IEEE International Symposium on Information Theory

[4] Amin O, Bedeer E, Ahmed M, Dobre O (to appear) Energy efficiency - spectral efficiency trade-off: A multiobjective optimization approach. IEEE Trans Veh Technol

[5] Andrews JG, Claussen H, Dohler M, Rangan S, Reed MC (Apr. 2012) Femtocells: Past, present, and future. IEEE J Sel Areas Commun 30(3):497-508

[6] Bajalinov EB (2003) Linear-Fractional Programming: Theory, Methods, Applications and Software, vol 84. Springer

[7] Ban TW, Choi W, Sung DK (Jun. 2009) Capacity and energy efficiency of multi-user spectrum sharing systems with opportunistic scheduling. IEEE Trans Wireless Commun 8(6):2836-2841

[8] Bansal G, Hossain M, Bhargava V (2008) Optimal and suboptimal power allocation schemes for OFDM-based cognitive radio systems. IEEE Trans Wireless Commun 7(11):4710-4718

[9] Bayhan S, Alagoz F (Feb. 2013) Scheduling in centralized cognitive radio networks for energy efficiency. IEEE Trans Veh Technol 62(2):582-595

[10] Bayhan S, Eryigit S, Alagoz F, Tugcu T (Jun. 2013) Low complexity uplink schedulers for energy-efficient cognitive radio networks. IEEE Commun Lett 2(3):363-366

[11] Bedeer E, Dobre OA, Ahmed MH, Baddour K (Apr. 2014) A multiobjective optimization approach for optimal link adaptation of OFDM-based cognitive radio systems with imperfect spectrum sensing. IEEE Trans Wireless Commun 13(4):2339-2351

[12] Bedeer E, Amin O, Dobre O, Ahmed M, Baddour K (Jun. 2015) Energyefficient power loading for OFDM-based cognitive radio systems with channel uncertainties. IEEE Trans Veh Technol 64(6):2672 - 2677

[13] Bertsekas DP, Bertsekas DP, Bertsekas DP, Bertsekas DP (1995) Dynamic programming and optimal control, vol 1. Athena Scientific Belmont, MA

[14] Boggs PT, Tolle JW (Jan. 1995) Sequential quadratic programming. Acta numerica 4:1-51

[15] Bolla R, Bruschi R, Davoli F, Cucchietti F (Second Quarter 2011) Energy efficiency in the future internet: a survey of existing approaches and trends in energy-aware fixed network infrastructures. IEEE Commun Surveys Tuts 13(2):223-244 
[16] Boyd S, Vandenberghe L (2004) Convex Optimization. Cambridge University Press

[17] Chen R, Park JM, Hou YT, Reed JH (Apr. 2008) Toward secure distributed spectrum sensing in cognitive radio networks. IEEE Commun Mag 46(4):5055

[18] Chen Y, Nossek J, Mezghani A (Aug. 2013) Circuit-aware cognitive radios for energy-efficient communications. IEEE Wireless Commun Lett 2(3):323-326

[19] Cheung WC, Quek TQ, Kountouris M (Apr. 2012) Throughput optimization, spectrum allocation, and access control in two-tier femtocell networks. IEEE J Sel Areas Commun 30(3):561-574

[20] Cui S, Goldsmith AJ, Bahai A (Sep. 2005) Energy-constrained modulation optimization. IEEE Trans Wireless Commun 4(5):2349-2360

[21] Dinkelbach W (Mar. 1967) On nonlinear fractional programming. Management Science 13(7):492-498

[22] Du H, Ratnarajah T (May 2013) Robust utility maximization and admission control for a MIMO cognitive radio network. IEEE Trans Veh Technol 62(4):1707-1718

[23] Ericsson (Aug. 2007) Sustainable energy use in mobile communications

[24] Fu L, Zhang YJA, Huang J (Nov. 2013) Energy efficient transmissions in MIMO cognitive radio networks. IEEE J Sel Areas Commun 31(11):24202431

[25] Gao S, Qian L, Vaman DR (Oct. 2009) Distributed energy efficient spectrum access in cognitive radio wireless ad hoc networks. IEEE Trans Wireless Commun 8(10):5202-5213

[26] Han JA, Jeon WS, Jeong DG (May 2011) Energy-efficient channel management scheme for cognitive radio sensor networks. IEEE Trans Veh Technol 60(4):1905-1910

[27] Hasan Z, Bansal G, Hossain E, Bhargava V (Dec. 2009) Energy-efficient power allocation in OFDM-based cognitive radio systems: A risk-return model. IEEE Trans Wireless Commun 8(12):6078-6088

[28] Hossain E, Bhargava V (2007) Cognitive Wireless Communication Networks. Springer

[29] Huang D, Kang G, Wang B, Tian H (May 2013) Energy-efficient spectrum sensing strategy in cognitive radio networks. IEEE Commun Lett 17(5):928931

[30] Huang S, Chen H, Zhang Y, Zhao F (Apr. 2012) Energy-efficient cooperative spectrum sensing with amplify-and-forward relaying. IEEE Commun Lett 16(4):450-453

[31] Liang YC, Zeng Y, Peh EC, Hoang AT (Apr. 2008) Sensing-throughput tradeoff for cognitive radio networks. IEEE Trans Wireless Commun 7(4):13261337

[32] Mahmoud H, Yucek T, Arslan H (2009) OFDM for cognitive radio: merits and challenges. IEEE Wireless Commun Mag 16(2):6-15 
[33] Maleki S, Pandharipande A, Leus G (Mar. 2011) Energy-efficient distributed spectrum sensing for cognitive sensor networks. IEEE Sensors J 11(3):565573

[34] Mao J, Xie G, Gao J, Liu Y (Feb. 2013) Energy efficiency optimization for cognitive radio MIMO broadcast channels. IEEE Commun Lett 17(2):337340

[35] Mao J, Xie G, Gao J, Liu Y (May 2013) Energy efficiency optimization for OFDM-based cognitive radio systems: A water-filling factor aided search method. IEEE Trans Commun 12(5):2366-2375

[36] Monahan GE (Jan. 1982) State of the art-a survey of partially observable markov decision processes: Theory, models, and algorithms. Management Science 28(1):1-16

[37] Nguyen D, Tran LN, Pirinen P, Latva-aho M (Aug. 2013) Precoding for full duplex multiuser MIMO systems: Spectral and energy efficiency maximization. IEEE Trans Signal Process 61(16):4038-4050

[38] Oto MC, Akan OB (Apr. 2012) Energy-efficient packet size optimization for cognitive radio sensor networks. IEEE Trans Wireless Commun 11(4):15441553

[39] Pei Y, Liang YC, Teh KC, Li KH (Sep. 2011) Energy-efficient design of sequential channel sensing in cognitive radio networks: optimal sensing strategy, power allocation, and sensing order. IEEE J Sel Areas Commun 29(8):16481659

[40] Sanchez SM, Souza RD, Fernández EMG, Reguera VA (May 2013) Rate and energy efficient power control in a cognitive radio ad hoc network. IEEE Signal Process Lett 20(5):451-454

[41] Shi Z, Teh K, Li K (Mar. 2013) Energy-efficient joint design of sensing and transmission durations for protection of primary user in cognitive radio systems. IEEE Commun Lett 17(3):565-568

[42] Sun X, Tsang D (Mar. 2013) Energy-efficient cooperative sensing scheduling for multi-band cognitive radio networks. IEEE Trans Wireless Commun 12(10):4943-4955

[43] Wang J, Palomar DP (Aug. 2009) Worst-case robust MIMO transmission with imperfect channel knowledge. IEEE Trans Signal Process 57(8):3086-3100

[44] Wang S, Ge M, Zhao W (Aug. 2013) Energy-efficient resource allocation for OFDM-based cognitive radio networks. IEEE Trans Commun 61(8):31813191

[45] Wang Y, Xu W, Yang K, Lin J (Sep. 2012) Optimal energy-efficient power allocation for OFDM-based cognitive radio networks. IEEE Commun Lett 16(9):1420-1423

[46] Wildemeersch M, Quek T, Slump C, Rabbachin A (Sep. 2013) Cognitive small cell networks: energy efficiency and trade-offs. IEEE Trans Commun 61(9):4016-4029

[47] Wu Y, Tsang DH (May 2011) Energy-efficient spectrum sensing and transmission for cognitive radio system. IEEE Commun Lett 15(5):545-547 
[48] Wu Y, Lau VK, Tsang DH, Qian LP (Sep. 2012) Energy-efficient delayconstrained transmission and sensing for cognitive radio systems. IEEE Trans Veh Technol 61(7):3100-3113

[49] Xiong C, Lu L, Li G (Mar. 2014) Energy-efficient spectrum access in cognitive radios. IEEE J Sel Areas Commun 32(3):550-562

[50] Yucek T, Arslan H (2009) A survey of spectrum sensing algorithms for cognitive radio applications. IEEE Commun Surveys Tuts 11(1):116-130

[51] Yue H, Pan M, Fang Y, Glisic S (May 2013) Spectrum and energy efficient relay station placement in cognitive radio networks. IEEE J Sel Areas Commun 31(5):883-893

[52] Zhang L, Liang YC, Xin Y (Jan. 2008) Joint beamforming and power allocation for multiple access channels in cognitive radio networks. IEEE J Sel Areas Commun 26(1):38-51 
Table 1 LIST OF SYMBOLS

\begin{tabular}{l|l}
\hline$\alpha$ & path loss exponent \\
\hline$\beta$ & cooperative sensing gain \\
\hline$\gamma_{n}$ & channel-to-interference-plus-noise ratio (CINR) of $n$th subcarrier \\
\hline$\gamma_{k, n}$ & CINR for the $n$th subcarrier used by the $k$ th SU \\
\hline$\Delta$ & step of the power control \\
\hline$\zeta$ & power amplifier efficiency \\
\hline$\eta_{\mathrm{EE}, \text { inst }}$ & instantaneous EE \\
\hline$\eta_{\mathrm{EE}, \text { inst }}^{\text {Wort }}$ & worst EE \\
\hline$\eta_{\mathrm{EC}, \text { inst }}$ & instantaneous energy consumption \\
\hline$\eta_{\mathrm{EC}, \text { av }}$ & average energy consumption \\
\hline$\eta_{\mathrm{gain}}$ & cooperative sensing gain per joule per SU \\
\hline$\eta_{\mathrm{EE}, \text { inst }}^{\text {inv }}$ & inverse of instantaneous EE \\
\hline$\eta_{\mathrm{EE}, \text { av }}^{\text {inv }}$ & inverse of average EE \\
\hline$\Lambda$ & bit error rate \\
\hline$\mu$ & SU sleep rate \\
\hline$\nu$ & censoring rate \\
\hline$\Phi_{\mathrm{m}}$ & density of mobile base station of the 1 st tier network \\
\hline$\sigma_{\mathrm{S}}$ & density of small cell access points of the 2 nd tier network \\
\hline$\rho_{k, n}$ & channel allocation indicator \\
\hline$\sigma_{\Delta \mathrm{H}}^{2}$ & minimum mean square error \\
\hline$\sigma_{n}^{2}$ & noise variance \\
\hline$\phi_{\mathrm{PU}}^{\text {idle }}$ & probability that PU is not using the channel \\
\hline$\phi_{\mathrm{PU}}^{\text {busy }}$ & probability that PU is using the channel \\
\hline$\phi_{\mathrm{fa}}$ & probability of false-alarm \\
\hline$\phi_{\mathrm{md}}$ & probability of mis-detection \\
\hline$\phi_{\mathrm{PU}}^{\text {re }}$ & probability that PU reoccupies the channel during the SU transmission \\
\hline$\phi_{n}$ & posterior probability that the PU channel $n$ is identified to be idle while it is truly idle \\
\hline$\phi_{\mathrm{UE}}$ & probability that there is a user equipment within the coverage of a small cell access points (SAP) unit \\
\hline$\phi_{\mathrm{S}}$ & success probability of a typical user within the coverage area of SAP \\
\hline$\phi_{n, \mathrm{fa}}$ & probability of false alarm of channel $n$ \\
\hline$\phi_{n}^{\text {idle }}$ & probability that channel $n$ is idle \\
\hline
\end{tabular}


Table 2 LIST OF SYMBOLS (CONT'D)

\begin{tabular}{|c|c|}
\hline$B$ & bandwidth \\
\hline$d_{\mathrm{PS}}$ & distance from the PU transmitter to the SU receiver \\
\hline$d_{\mathrm{SS}}$ & distance from the SU transmitter to the SU receiver \\
\hline$E_{k, n}$ & energy consumed by the $k$ th user to employ the $n$th channel \\
\hline$E_{k}^{\text {idle }}$ & energy consumed by the $k$ th user while being in idle state \\
\hline$E^{\mathrm{se}}$ & energy consumed by all the cooperative sensing SUs \\
\hline$E^{\mathrm{tr}}$ & energy consumed during the transmission \\
\hline$E_{\mathrm{c}}$ & consumed energy \\
\hline$G$ & path loss \\
\hline$\hat{H}_{n}$ & estimate of the complex channel gain on subcarrier $n$ \\
\hline $\mathscr{J}_{n}$ & interference from all PUs to subcarrier $n$ \\
\hline$K$ & total number of SUs \\
\hline$K^{\text {relay }}$ & number of relays \\
\hline$K^{\mathrm{se}}$ & number of sensing users \\
\hline $\mathscr{L}\left(p_{n}\right)$ & loss function of power consumed in subcarrier $n$ \\
\hline$\ell$ & packet length \\
\hline$n$ & subcarrier index \\
\hline$N$ & total number of subcarriers \\
\hline$N_{\text {samples }}$ & total number of samples \\
\hline$p_{n}$ & power allocated to subcarrier $n$ \\
\hline$p_{c}$ & circuitry power consumption \\
\hline$p_{k, n}$ & power allocated to the $n$th subcarrier employed by the $k$ th SU \\
\hline$p^{\mathrm{se}}$ & sensing power \\
\hline$p^{\text {tr }}$ & transmission power \\
\hline$p_{\mathrm{SU}}$ & SU transmit power \\
\hline$p^{\mathrm{PA}}$ & power consumed in the power amplifier \\
\hline$p^{\mathrm{LNA}}$ & power consumed in low noise amplifier \\
\hline$p^{\mathrm{ADC}}$ & power consumed in the ADC \\
\hline$p^{\mathrm{syn}}$ & synchronization power \\
\hline$p^{\text {proc }}$ & processing power \\
\hline$p^{\text {relay }}$ & average transmit power of a given relay \\
\hline $\mathbf{Q}_{k}$ & covariance matrix of $k$ th SU \\
\hline$r_{k, n}$ & rate of the $k$ th user employing the $n$th channel \\
\hline$r$ & transmission rate \\
\hline$r_{k}$ & rate of $k$ th SU \\
\hline$t$ & total frame duration \\
\hline$t_{k, n}^{\mathrm{cs}}$ & time required by the $k$ th user to tune to the $n$th channel \\
\hline$t^{\mathrm{se}}$ & sensing duration \\
\hline$t^{\mathrm{tr}}$ & transmission duration \\
\hline$T$ & is the total slot length \\
\hline$T_{\text {samples }}$ & sampling period \\
\hline$U_{\text {rate-loss }}$ & rate-loss utility metric \\
\hline$U_{\text {rate-power }}$ & rate-power utility metric \\
\hline$U_{\text {rate-energy }}$ & rate-energy utility metric \\
\hline$U_{\text {energy-reliability }}$ & energy-reliability utility metric \\
\hline$w_{k}$ & relative weight of the EE of the $k$ th SU \\
\hline
\end{tabular}

